\title{
BMJ Open Sensitivity and specificity of different imaging modalities in diagnosing necrotising enterocolitis in a Polish population of preterm infants: a diagnostic test accuracy study protocol
}

\author{
Joanna Seliga-Siwecka (D) , ${ }^{1}$ Jakub Rutkowski, ${ }^{2}$ Wojciech Margas, ${ }^{2}$ \\ Joanna Puskarz- Gąsowska, ${ }^{1}$ Renata Bokiniec ${ }^{1}$
}

To cite: Seliga-Siwecka J, Rutkowski J, Margas W, et al. Sensitivity and specificity of different imaging modalities in diagnosing necrotising enterocolitis in a Polish population of preterm infants: a diagnostic test accuracy study protocol. BMJ Open 2020;10:e033519. doi:10.1136/ bmjopen-2019-033519

- Prepublication history and additional material for this paper are available online. To view these files, please visit the journal online (http://dx.doi org/10.1136/bmjopen-2019033519).

Received 27 August 2019 Revised 09 March 2020 Accepted 12 May 2020
Check for updates

(C) Author(s) (or their employer(s)) 2020. Re-use permitted under CC BY-NC. No commercial re-use. See rights and permissions. Published by BMJ.

${ }^{1}$ Neonatal and Intensive Care Department, Medical University of Warsaw, Warsaw, Poland ${ }^{2}$ HTA Consulting, Cracow, Poland

Correspondence to

Dr Renata Bokiniec;

renata.bokiniec@wum.edu.pl

\section{ABSTRACT}

Introduction Necrotising enterocolitis (NEC) is one of the most serious conditions in newborn infants, affecting up to $10 \%$ of very low birth weight (VLBW) infants. Mortality rates can rise as high as $60 \%$.

The suspected diagnosis is confirmed with typical findings on abdominal radiography (AR) such as pneumatosis intestinalis (PI), portal vein gas (PVG) and in extreme cases pneumoperitoneum. Abdominal ultrasound (AUS) can depict PI, PVG and pnuemoperitoneum (in some cases ahead of AR), but it also provides other crucial information such as bowel wall viability (thickness or thinning) and free abdominal fluid. These additional findings are helpful in diagnosing and managing NEC.

Methods and analysis The hypothesis being tested is that preforming an AR in patients with clinical symptoms of NEC, but inconclusive/normal AR will enhance detection rates, and expedite treatment in infants born at $<32$ weeks. Additionally, the time needed to initiate treatment, according to decision made based on AR or AR and AUS will also be compared. The use of AUS together with AR as an add-on test may increase the accuracy of diagnosing NEC and expedite life-saving treatment. We plan to recruit 200 VLBW infants, who are most prone to NEC. It will also be the first multicentre study evaluating the use of AUS as an add-on test, enabling us to recruit a significantly higher number of patients compared with published studies. Ethics and dissemination The Bioethical Committee of the Medical University of Warsaw has approved the study (KB 130/2017). We plan to submit our findings to international peer-reviewed journals. Abstract will be submitted to local and international conferences. Trial registration number NCT03188380; Protocol version: V.2.08.2019; Pre-results.

\section{INTRODUCTION}

Necrotising enterocolitis (NEC) is one of the most serious conditions in neonates, affecting up to $10 \%$ of very low birth weight (VLBW) infants. In the most premature population mortality rates can rise as high as $60 \%{ }^{1}$

\section{Strengths and limitations of this study}

- This will be the first multicentre comparative study, designed to focus on data from verylow birth weight infants, who are most prone to necrotising enterocolitis (NEC).

- We plan to assess the diagnostic value of abdominalultrasound (AUS) as an add-on test in decisionmaking regarding management and treatment of NEC.

- A multicentre study will allow us to recruit a significantly higher number of patients as compared with published studies.

- AUS definite criteria of NEC are not established.

- Intraobserver and interobserver variability is possible when diagnosing NEC based on ultrasonography.

In 1978 Bell developed a staging system for NEC, which was then modified by Walsh and Kliegman in the mid 1980's. ${ }^{2}{ }^{3}$ This widely adopted classification is based solely on plain abdominal radiography (AR), despite first reports on ultrasound use for diagnosing NEC being published in the early 1980 's. ${ }^{4-6}$ The suspected diagnosis is confirmed with typical findings on AR such as pneumatosis intestinalis $(\mathrm{PI})$, portal vein gas (PVG) and in extreme cases pneumoperitoneum ${ }^{7-9} \mathrm{~A}$ combination of clinical symptoms and AR findings allows grading of interventions and standardised treatment. Serious NEC can present without PI or PVG, especially in cases with very high abdominal pressure or intestinal loop overlap.

Abdominal ultrasound (AUS) can depict PI, PVG and pneumoperitoneum (in some cases ahead of AR), but it also provides additional crucial information such as bowel wall variability (thickness $>2.7 \mathrm{~mm}$ or thinning $<1 \mathrm{~mm}$ ) and presence of free abdominal 
fluid. ${ }^{5}$ Doppler evaluation is essential to assess absent or increased perfusion. Published reports have emphasised the role of the bowel wall thickness, peristalsis and bowel wall perfusion (BWP) as findings typical for NEC and solely detected on AUS. ${ }^{10}$

A non-systematic literature in MEDLINE and PubMed of seven key phrases (necrotising enterocolitis, preterm, very low birth weight infant, neonate, ultrasound, radiography) and personal attendance to three recent conferences on closely related topics produced a limited number of retrospective cohort studies and two prospective reports, evaluating the use of AUS in diagnosing NEC. We only found two registered protocols of small prospective studies on ClinicalTrials.gov.

We chose to evaluate the use of AUS (index test) in infants born at $<32$ weeks of gestation. In this population a combination of genetic predisposition, intestinal prematurity, an imbalance in microvascular tone together with abnormal microbial colonisation (due to the use of broad-spectrum antibiotics and delayed enteral feeding) along with a highly immunoreactive intestinal microflora lead to an increased risk of developing NEC. ${ }^{11}$

Despite clearly defined pathognomonic symptoms such PI and PVG on AR, diagnosing NEC still remains a challenge. In a significant group of VLBW infants, clinical symptoms are not always confirmed by traditional imaging methods. In these neonates AUS has been found to be more accurate in detecting not only absent bowel perfusion and bowel necrosis, but also traditional radiological features such as PI and pneumoperitoneum. ${ }^{12-14}$ AUS can be performed repeatedly at the patient's bedside, avoids radiation exposure and allows to follow the dynamic process of NEC. $^{15}$

In spite of variable sensitivity and specificity, AR still remains the gold standard for confirming clinical symptoms. ${ }^{15}$ Its selection as a reference test is therefore justifiable. PI and/or PVG are detected on AR are pathognomonic for NEC. ${ }^{11}$
The use of AUS together with AR as an add-on test may increase the accuracy of diagnosing NEC and expedite treatment. Swift implementation of antibiotics and bowel rest is extremely important. If left untreated, NEC leads to bowel perforation requiring surgical intervention, complicated with high morbidity rates. ${ }^{16}{ }^{17}$ The annual costs of caring for affected infants in the USA are between $\$ 500$ million to $\$ 1$ billion. This data is not available for the Polish or European population, but we assume it is comparable to American reports. The need for bowel resection is one of the most common causes of short bowel syndrome in the paediatric population.

Out of the published studies only two have evaluated all possible features detected AUS. These retrospective reports were small in numbers and did not discrepant between term and preterm infants. ${ }^{10} 18$

To our best knowledge, our study will be the first multicentre study focussing on VLBW infants with a significantly higher number of patients compared with published studies.

We hope that our results will shed a new light on the use of AUS in VLBW infants and how its findings should be interpreted.

\section{Study objectives and hypothesis}

The hypothesis being tested is that preforming an AUS in patients with clinical symptoms of NEC, but inconclusive/normal AR will enhance NEC detection rates and expedite treatment.

The secondary objective of this study is to evaluate the sensitivity and specificity of a diagnostic strategy involving a combination of AR (reference test) followed by AUS (index test) as compared with AR (reference test) in diagnosing NEC.

As suggested by Woolf in 1991, while preparing the research hypothesis we created an analytical framework in order to visually clarify the relationship between the hypothetical healthcare intervention and outcomes, and to guide us in any future review process (figure 1.). ${ }^{19} 20$

Figure 1 Analytical framework for the evaluation of abdominal ultrasound and plain radiography. 


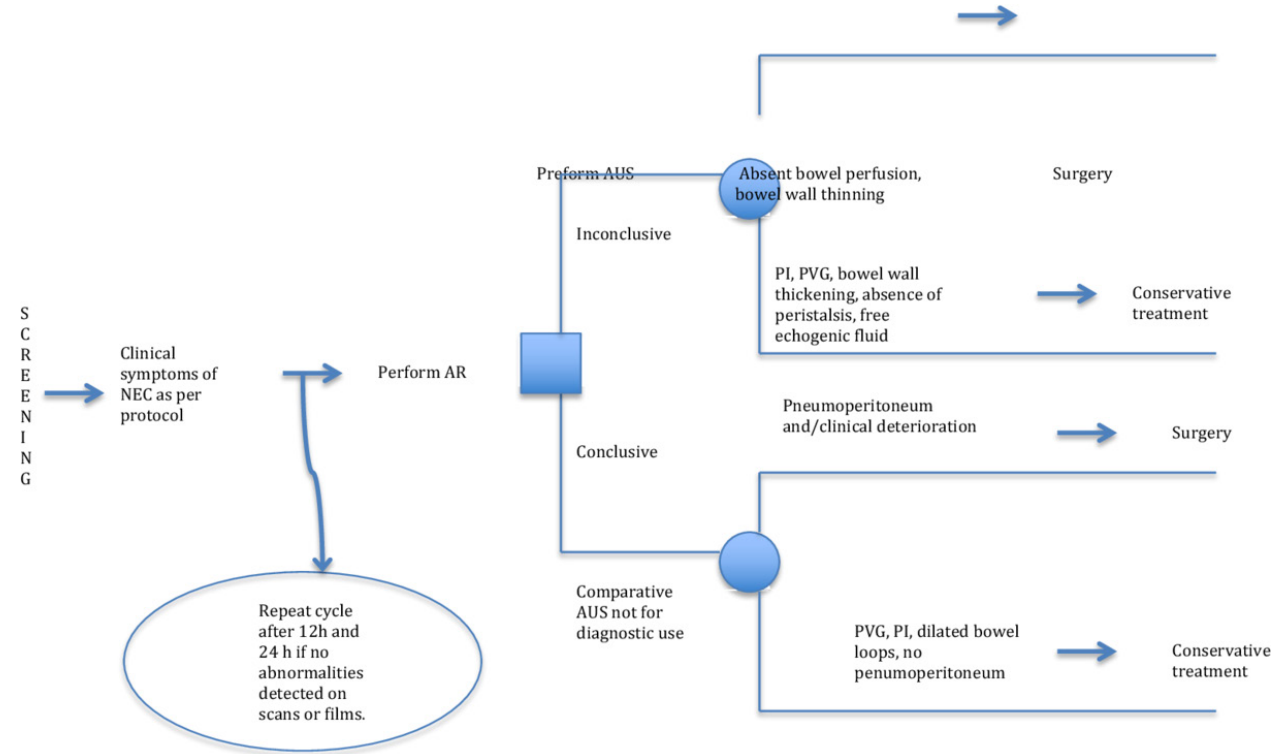

Figure 2 Diagnostic decision tree for AUS as an add-on test. AUS, abdominal ultrasound; AR, abdominal radiograph; NEC, necrotising enterocolitis; PVG, portal vein gas; PI, pneumatosis intestinalis.

In order to determine how our test strategy may affect intermediate and clinical outcomes we designed a decision tree model (figure 2.).

\section{METHODS}

\section{Eligibility criteria}

In this single-gate study we plan to include all consecutive preterm infants (born at $<32$ weeks of gestation) with suspected NEC based on the presence of at least three of the following clinical symptoms: ${ }^{311} 2122$

- Abdominal distension (abdominal circumference increase $>2 \mathrm{~cm}$ over 12 hours) .

- Visible bowels loops.

- Feeding intolerance (defined as one of the following; bilious residuals or bilious emesis $\geq 2$ consecutive feeds, or pre-feed gastric residual volume of $>50 \%$ in $\geq 2$ consecutive feeds).

- Temperature instability (defined as $\geq 2$ consecutive measurements within 2 hours).

- Frank bloody stools.

- Cardiovascular instability (hypotension; defined as mean arterial pressure $<30 \mathrm{~mm} \mathrm{Hg}$, and/or tachycardia $>160$ beats per minute or bradycardia $<80$ beats per minute greater than three episodes per hour requiring manual stimulation of bag and mask ventilation).

- Recurrent apnoea.

- Increase of abdominal girth $>2 \mathrm{~cm}$ (allowing interobserver variability of $1 \mathrm{~cm}$ ) within 12 hours.

- Abdominal wall erythema.

And at least two of the below laboratory findings: ${ }^{3}$

- Thrombocytopenia $<50 \times 10^{3} / \mathrm{uL}$.

- Leucopenia $<6 \times 10^{6} / \mathrm{uL}$.

- C-reactiveprotein $>10 \mathrm{mg} / \mathrm{L}$.

- Procalcitonincoagulation factors $>1 \mathrm{ng} / \mathrm{mL}$.
- Coagulopathy.

The attending physician, based on eligibility criteria, will determine participants. $\mathrm{He} /$ she will then immediately notify the research team. An AR will be performed within $30 \mathrm{~min}$ of symptoms presentation. If findings, typical for NEC, are recorded on AR, the patient will receive appropriate treatment. ${ }^{3}$ If the AR is inconclusive or no abnormalities are detected, it will be followed by an AUS not later than 60 min post AR. Decisions regarding treatment will be only based on clinical symptoms and $\mathrm{AR}^{3}$ In cases requiring surgical consultation, the surgeon will be informed about AUS findings.

If none of the trained ultrasonographers are available in due course, the PI will make an executive decision to exclude the patient.

\section{Exclusion criteria}

- < 23 weeks of gestational age or $>32$ weeks (estimated by ultrasound during first trimester screening).

- Congenital abnormalities.

- No parental consent.

The study will take place at four level III perinatal centres.

All consecutive infants presenting with clinical symptoms of NEC, and whose parents provided written consent for participating in the trial, will be screened for eligibility.

\section{Data collection}

The study will be performed in two phases. Phase one will consist of a prospective observational study. Data collected in phase one during the second phase of the study. During the observational period all participants will have AR and AUS performed (if clinical symptoms of NEC are present) but the decision regarding treatment will be based on AR and clinical findings. Collected data 
will be used during phase two in order to evaluate AUS as an add-on test; the diagnosis and treatment decision will be made by blinded to real data outcomes investigators. Each blinded radiologist will be presented with AR findings and clinical symptoms, or AR+AUS findings and symptoms. Based on the provided data the radiologist will diagnose or exclude NEC. Diagnosis and treatment decisions from real data (phase one) and phase two will be compared with to evaluate the sensitivity and specificity of AUS as an add-on test. Questionnaire details are provided in online supplementary appendix 1 .

\section{Staff training}

Prior to recruitment commencement, a meeting will be scheduled in each centre to introduce the study to unit staff. This session will include:

- Brief presentation on study aims and eligibility criteria.

- Introduction to oral and written informed consent process.

- Presentation of data collection platform and its requirements.

- NEC diagnosis guidelines for radiologists and ultrasonographists.

A subsequent meeting will take place 2 weeks after initiating the study. Staff will be asked about any problems they might be experiencing with implementing the study; such as patient recruitment, data collection and NEC diagnosis.

Multicentre research meetings will be held two times a year for the first 2 years in order to improve adherence to study protocol, improve recruitment rates and resolve on-going issues. For the following 2 years, meetings will be held every 1 month to analyse results and resolve any data collection issues.
Index test

AUS will be performed using devices presented in table 1 . The examination will take approximately $15 \mathrm{~min}$ and will be divided into two parts. The examination will be stopped if any of the following changes in vital signs and oxygen saturation levels occur; heart rate less than 100 or more than 200 beats per minute and oxygen saturation less than $90 \%$. Initially, high-resolution micro convex transducers ( 5 to $12 \mathrm{MHz}$ ) will be used due to the fine texture of anatomical structures. PVG, gall bladder, free fluid and free abdominal air will be screened with a small convex-array transducer, ${ }^{15}$ followed by bowel evaluation with a linear array transducer ( 5 to $17 \mathrm{MHz}$ ). All four abdomen quadrants will be examined in a systematic manner.

Based on available literature we have adopted the following definitions for AUS findings.

1. Grey-scale ultrasound evaluation will be used to assess bowel wall echogenicity, measure bowel wall thickness, peristalsis, PI, PVG, free abdominal air and fluid. ${ }^{15}$

a. Bowel wall thickness of 1.1 to $2 \mathrm{~mm}$ is considered as normal. A bowel wall thickness of $>2.7 \mathrm{~mm}$ accompanied by an increase in echogenicity will be considered as suspicious. ${ }^{1015}$

b. Bowel wall thickness below $1.0 \mathrm{~mm}$ will be considered as abnormal thinning as a result of ischaemia or necrosis. Measurements will be recorded in relaxed bowel segments.

c. PI will be defined as small intramural hyperechoic focusses in the bowel wall, which will not change position despite peristalsis, respiratory movement or abdominal compression with the transducer.

d. Normal peristaltic contractions of the small bowel will be defined as more than 10 movements/min.

Table 1 Enrolment, intervention and assessment timetable

\begin{tabular}{|c|c|c|c|c|}
\hline & \multicolumn{4}{|c|}{ Study period } \\
\hline & Enrolment & T1 and T2 & Post-inclusion & \\
\hline TIMEPOINT & $0-7$ days & $>7$ days & Discharge or 40 weeks PCA & 52 weeks PCA \\
\hline \multicolumn{5}{|l|}{ ENROLMENT } \\
\hline Eligibility screen & $x$ & $x$ & & \\
\hline Informed consent & $\mathrm{X}$ & & & \\
\hline Blood sampling ${ }^{\star}$ & & $\mathrm{X}$ & & \\
\hline (Abdominal ultrasound) & & $x$ & & \\
\hline (Abdominal radiograph) & & $\mathrm{X}$ & & \\
\hline Anthropometric measurements & & & X & $x$ \\
\hline
\end{tabular}

T1- index test performance.

T2 - reference test performance.

F1 - follow-up assessment at discharge or 40 weeks PCA.

F2 - follow-up assessment at 52 weeks PCA.

PCA- post conceptional age.

*Blood sampling for complete blood count.

CRP, C-reactive protein; NEC, necrotising enterocolitis; PCT, procalcitonin coagulation factors. 
e. For evaluation of PVG, the portal vein will be adjusted in a transverse or longitudinal section of the liver. PVG will be defined as highly echogenic round particles with a diameter of approximately $1 \mathrm{~mm}$ registered within portal circulation.

f. Using a transverse section of the right upper abdomen showing the liver just below the diaphragm, free abdominal gas will be detected as a bright echogenicity between the abdominal wall and the anterior surface of the liver.

g. Free abdominal fluid will be evaluated as an hypoechogenic material between the bowel loops.

2. Colour Doppler (CD) ultrasound will be required to evaluate blood flow in the superior mesenteric artery (SMA) and BWP.

a. To assess the SMA flow the transducer will be held in the longitudinal section just below the xiphoid. Elevated systolic velocities (up to 100 to $120 \mathrm{~cm} / \mathrm{s}$ ) and high diastolic velocity will be suspicious for NEC.

b. Evaluation of BWP; three categories of flow in the bowel wall will be recognised in colour Doppler: normal ( 1 to $9 \mathrm{CD}$ signal dots per $\mathrm{cm}^{2}$ ), increased ('zebra' pattern, 'Y' pattern or 'ring' pattern) and absent (when no colour Doppler signals will be present) which will be reported as transmural necrosis.

Ultrasonographer experience will vary between centres ( 5 to 20 years of experience). All examinations will be performed at the bedside and saved on a secure webbased platform. No specific pre-test requirements will be required. The results of the examinations will be immediately reported on a secure website. In order to decrease observer bias, each ultrasound and plain radiology film will be labelled with a unique study number. AR and/ or AUS studies will re-assessed bi-monthly by a radiologist from one of the participating centres other than the patients referring centre. The re-assessors will be blinded to patient symptoms and outcomes.

\section{Reference test}

Plain AR is the currently only gold standard for evaluating neonates with symptoms suggesting NEC and will be used as the reference test. The disease tends to progress rapidly, hence serial assessments (every 12 to 24 hours) are recommended. ${ }^{23}$

Each time an AR is performed one image will be obtained with a vertical beam and a horizontal beam, with the patient supine. This will allow avoiding moving the sick newborn.

The following radiography findings are considered typical for NEC:

- Intramural gas (pneumatosis intestinalis) may precede clinical symptoms. ${ }^{24}$ Despite being present in other diseases, PI is considered to be pathognomonic for NEC. ${ }^{25-27}$ The incidence in reported studies varies from $19 \%$ to $98 \% .{ }^{24527}$ However, in the presence of clinical symptoms, lack of PI does not exclude NEC. ${ }^{27}$ PI is most commonly present in the distal small bowel and large bowel, hence it is usually seen in the right lower quadrant, although it may be present in any part of the gastrointestinal tract. ${ }^{25}$ On AR it will present as diffuse or localised linear or rounded radiolucencies. It is important to differentiate PI form overlapping loops. Visible white lines often accompany the black lines of intramural gas. The white lines represent, lifted by gas, mucosa and submucosa. ${ }^{23}$

- Portal venous gas is an extension of intramural gas present in the bowel wall veins, passing into the portal vein system. It is usually present in up to $30 \%$ of patients with NEC. ${ }^{28}{ }^{29}$ On supine AR it appears as branching, linear, radiolucent vessels, which may extend toward the periphery of both hepatic lobes. However, it is not as early a sign as PI.

- Free intraperitoneal gas is a result of bowel perforation, which usually is located in the distal ileum and proximal colon. It is the only universally accepted radiologic indication for surgical intervention. ${ }^{12}$ Plain AR is the standard method for detecting free gas. A crosstable later view with a horizontal view is recommended. Free gas may appear as triangular lucencies between loops of bowel anteriorly, just beneath the abdominal wall or as small bubbles or linear gas collections anterior to the liver. ${ }^{30}$ On the supine view, large amounts of gas may give the 'football; sign (the gas outlines the whole of the peritoneal cavity, the under surface of the diaphragm and the falciform ligament) ${ }^{23}$

- Bowel wall pattern in NEC usually presents as nonspecific small bowel dilatation, and can be appreciated on the plain AR. ${ }^{24}$

Available studies have focussed on evaluating the validity and the prognostic value of single findings rather a combination of symptoms. We have decided to evaluate a combination of findings, choosing those with the best prognostic values for NEC. ${ }^{15}$ We will define AUS as positive for $N E C$ when three of the following findings are appreciated on examination:

a. Abnormal bowel wall thickness $(<1.0 \mathrm{~mm}$ or $>2.7 \mathrm{~mm})$. b. PI.

c. Free abdominal fluid as a focal fluid collection.

d. Abnormal SMA blood flow or bowel wall perfusion.

And one of the listed findings:

a. Free abdominal air.

b. Delayed peristalsis.

c. PVG.

AR will be classified as positive for NEC, if one of the following findings will be present: ${ }^{23}$

- Intramural gas (pneumatosis intestinalis).

- Portal venous gas.

- Free intraperitoneal gas.

And

- Small bowel dilatation.

Images of all patients will be reviewed twice throughout the study. Initial evaluations will be performed if the patient presents for symptoms suspicious for NEC. A radiologist with $>10$ years of experience, who will be blinded to all patient information, will read the AR. A neonatologist with (5, 10 or 20 years of experience, respectively) 
will perform the AUS. He/she will be blinded to all patient information. Since the AUS will be performed at the bedside, all vital signs will be sealed. Considering the nature of the AUS we will be unable to completely blind the examiner (the patient will be visible to the examiner). Each plain radiography and ultrasound file will be labelled with a unique study number and stored on a secured web-platform. The assessors of the reference standard will not have access to the index test results and vice versa.

\section{Primary objective}

To determine if AUS as an add-on to AR, performed on children with suspected NEC according to clinical criteria leads to additional diagnoses of NEC cases when the diagnosis is not confirmed based only on abdominal AR.

\section{Secondary objective}

Children initially diagnosed with suspected NEC based on clinical criteria, but not confirmed on AR will be reassessed with AR and AUS at 6, 12 and 24 hours of follow-up.

\section{Statistical analysis}

The discordance between diagnostic decision made based on AR only and AR and AUS will be compared with twotailed McNemar's test with paired measures. Differences in diagnosis (detection of NEC, with/without perforation and perforation without NEC symptoms) together with treatment decisions (surgery or conservative treatment) will be measured.

Conditional specificity and positive predictive values will be presented as a percentage, with $95 \%$ CIs.

The time to initiate treatment, according to decision made based on AR or AR and AUS will be compared using regression analysis.

Continuous (quantitative) variables will be presented as means, SD, medians, minima and maxima, while nominal (qualitative) variables will be presented as frequencies. Variables recorded at final physical examination will be compared with those obtained during enrolment using paired Student's t-test or Wilcoxon (in case of nonnormality) test for quantitative variables and marginal homogeneity test for qualitative variables.

A series of sensitive analysis will be made for all inconclusive detections (for real data, blinded AR and blinded $\mathrm{AR}$ and AUS).

We do not plan an interim analysis during the study period.

\section{Management of indeterminate and missing data for index test or reference standard results \\ In cases when diagnosis based on $\mathrm{AR}$ or $\mathrm{AR}+\mathrm{AUS}$ records will not be determined by the reader, the second blinded reader will be asked for the assessment, and the diag- nostic decision will be reached by consensus. Any subject with missing RT or AUS will be excluded, no missing data replacement will be provided.}

\section{Intended sample size and how it was determined}

No published data has been found regarding the specificity of ultrasound as an add-on diagnostic test in children with suspected, but not confirmed NEC. Thus, for the sample size estimation, $5 \%$ discordance between AUS and AR diagnostic decisions will be assumed as a clinically plausible difference.

Using a sample size assessment for two-sided equality McNemar's Z-test for contingency table with paired measures, to detect $5 \%$ discordance between AUS add-on versus $A R$ test with two-sided significance level and $90 \%$ power, 200 children will be enrolled in the study.

\section{Participant timeline and diagnostic decision tree}

All parents of infants born at less than 32 weeks of gestation and admitted to the participating units will be screened for eligibility criteria after 7 days of life. When screened positive, each participating neonate, will initially have an AR performed. If the reference test is conclusive for NEC, appropriate treatment will be established. However, in each case a simultaneous AUS will be performed, but will not be considered in the decision process. We plan to reassess patients at 6,12 and 24 hours even if the reference tests is found negative, as NEC may progress rapidly (figure 2).

\section{Recruitment}

A member of the research team in each centre will screen patients on admission and inform parents or caregivers about the study within the first 7 days of life. Care providers will receive both written and oral information about study inclusion criteria and main outcomes. All neonates will be routinely screened for eligibility criteria when presenting with symptoms suggestive of NEC, until the target population is achieved (200 subjects). We have not set a target recruitment rate per centre. All sites will follow the same recruitment strategy. The enrolment period will extend over 24 months.

\section{Monitoring}

Harms

We will define an adverse event (AE) as any untoward medical occurrence in a subject without regard to the possibility of a causal relationship. AE will be collected after the subject has provided consent and enrolled in the study. All AE occurring after entry into the study and until hospital discharge will be recorded. An AE that meets the criteria for a serious $\mathrm{AE}$ (SAE) between study enrolment and hospital discharge will be reported to the local Ethical Committee. A SAE for this study is any untoward medical occurrence that is believed by the investigators to be causally related to study-intervention and results in any of the following: Life-threatening condition (that is, immediate risk of death); severe or permanent disability and prolonged hospitalisation. SAE occurring after a subject is discontinued from the study will NOT be reported unless the investigators feel that the event may have been caused by the study drug or a protocol procedure. 


\section{Consent or assent}

All parents of infants born at less than 32 weeks of gestation and admitted to participating centres will be approached by one of the research team members within the first 7 days of life (as NEC usually presents after the first week of life). $\mathrm{He} / \mathrm{she}$ will provide oral and written information about the study. Patients will then be able to have an informed discussion with the attending physician. Research team members will obtain written consent from patients willing to participate in the trial. Information consent forms and information sheets will only be provided in Polish for all parents.

\section{Confidentiality}

Complete patient and study information will be stored on a secure, password protected, web-based platform. Only researchers involved in the study will be provided with a personalised login and password to access the study information. The statistical team will not have access to sensitive data such as date of birth, address and contact details. All records relevant medical history, together with copies of ultrasound clips and radiology reports will be stored separately in a locked file cabinet.

\section{Data sharing and management plan}

An electronic casereport form (CRF) will be used for this study. For each subject enrolled, a CRF must be completed by the investigator or a designated sub-investigator. This also applies to those subjects who fail to complete the study. If a subject withdraws from the study, the reason must be noted on the CRF. CRFs are to be completed on an ongoing basis. CRF entries and corrections will only be performed by study site staff, authorised by the investigator. Checks for possible errors and inconsistencies in the entered data will be carried out periodically.

All de-identified data collected during the trial will be available. The study protocol will also be available. These documents will be accessible to anyone who provides a methodologically sound proposal immediately following publication with no end date.

\section{Patient and public involvement}

We did not involve parents of our patients or the public in the development of the research question, outcome measures or study design. We do not plan to include patients in the recruitment and conduct of the study. After completing the study, we will present our results to parents during our units annual neonatal intensive care unit (NICU) Graduates Day.

\section{Implications for practice}

AUS can be performed repeatedly at the patient's bedside, avoids radiation exposure and allowing to follow the dynamic process of NEC. ${ }^{15}$ We hypothesise that the use of AUS together with AR as an add-on test will aid the physician in diagnosing NEC and expedite treatment. Swift implementation of antibiotics and bowel rest is extremely important. A delay in diagnosis leads to bowel perforation requiring surgical intervention, complicated with high morbidity rates. ${ }^{16}$ The need for bowel resection is one of the most common causes of short bowel syndrome in the paediatric population. ${ }^{17}$

We hope that our results will shed a new light on the use of AUS in VLBW infants and how its findings should be interpreted, in the most vulnerable population of preterm infants.

\section{ETHICS AND DISSEMINATION POLICY}

The Bioethical Committee of the Medical University of Warsaw has approved the study (KB 130/2017). All significant modifications in the protocol will be reported to the committee. We plan to submit our findings to international peer-reviewed journals and conferences (paediatric, surgical and radiology).

Collaborators The NECUS study group: Urszula Majewska, Agnieszka Ochoda, Piotr Kruczek, Piotr Szymański, Andrzej Grudzień, Agnieszka Szadkowska, Krystyna Potocka and Dawid Szpecht.

Contributors JSS, WM, JR and RB conceptualised the study. JSS wrote the first draft of the protocol. RB and JPG (Joanna Puskarz-Gąsowska) critically reviewed the protocol and accepted the final manuscript as submitted. All authors read and approved the final version.

Funding This work is supported by The European Structural and Investments Fund, grant number RPMA.01.02.00-14-b455/18.

Competing interests None declared.

Patient consent for publication Not required.

Provenance and peer review Not commissioned; externally peer reviewed.

Open access This is an open access article distributed in accordance with the Creative Commons Attribution Non Commercial (CC BY-NC 4.0) license, which permits others to distribute, remix, adapt, build upon this work non-commercially, and license their derivative works on different terms, provided the original work is properly cited, appropriate credit is given, any changes made indicated, and the use is non-commercial. See: http://creativecommons.org/licenses/by-nc/4.0/.

ORCID iD

Joanna Seliga-Siwecka http://orcid.org/0000-0002-4628-0541

\section{REFERENCES}

1 Clark RH, Gordon P, Walker WM, et al. Characteristics of patients who die of necrotizing enterocolitis. J Perinatol 2012;32:199-204.

2 Bell MJ, Ternberg JL, Feigin RD, et al. Neonatal necrotizing enterocolitis. therapeutic decisions based upon clinical staging. Ann Surg 1978;187:1-7.

3 Walsh MC, Kliegman RM. Necrotizing enterocolitis: treatment based on staging criteria. Pediatr Clin North Am 1986;33:179-201.

4 Malin SW, Bhutani VK, Ritchie WW, et al. Echogenic intravascular and hepatic microbubbles associated with necrotizing enterocolitis. $J$ Pediatr 1983;103:637-40.

5 Merritt CR, Goldsmith JP, Sharp MJ. Sonographic detection of portal venous gas in infants with necrotizing enterocolitis. AJR Am J Roentgenol 1984;143:1059-62.

6 Kodroff MB, Hartenberg MA, Goldschmidt RA. Ultrasonographic diagnosis of gangrenous bowel in neonatal necrotizing enterocolitis. Pediatr Radiol 1984;14:168-70.

7 Stiennon OA. Pneumatosis intestinals in the newborn. AMA Am J Dis Child 1951;81:651-63.

8 Wolfe JN, Evans WA. Gas in the portal veins of the liver in infants; a roentgenographic demonstration with postmortem anatomical correlation. Am J Roentgenol Radium Ther Nucl Med 1955;74:486-8.

9 Miller RE. Perforated viscus in infants: a new roentgen sign. Radiology 1960;74:65-7.

10 Faingold R, Daneman A, Tomlinson G, et al. Necrotizing enterocolitis: assessment of bowel viability with color Doppler us. Radiology 2005;235:587-94. 
11 Neu J, Walker WA. Necrotizing enterocolitis. N Engl J Med 2011;364:255-64.

12 Buonomo C. The radiology of necrotizing enterocolitis. Radiol Clin North Am 1999;37:1187-98. vii.

13 Dilli D, Suna Oğuz S, Erol R, et al. Does abdominal sonography provide additional information over abdominal plain radiography for diagnosis of necrotizing enterocolitis in neonates? Pediatr Surg Int 2011;27:321-7.

14 McBride WJ, Roy S, Brudnicki A, et al. Correlation of complex ascites with intestinal gangrene and perforation in neonates with necrotizing enterocolitis. J Pediatr Surg 2010;45:887-9.

15 Bohnhorst B. Usefulness of abdominal ultrasound in diagnosing necrotising enterocolitis. Arch Dis Child Fetal Neonatal Ed 2013;98:F445-50.

16 Fitzgibbons SC, Ching Y, Yu D, et al. Mortality of necrotizing enterocolitis expressed by birth weight categories. J Pediatr Surg 2009;44:1072-6. discussion 75-6.

17 Spencer AU, Kovacevich D, McKinney-Barnett M, et al. Pediatric short-bowel syndrome: the cost of comprehensive care. Am J Clin Nutr 2008;88:1552-9.

18 Silva CT, Daneman A, Navarro OM, et al. Correlation of sonographic findings and outcome in necrotizing enterocolitis. Pediatr Radiol 2007;37:274-82.

19 Matchar DB. Introduction to the Methods Guide for Medical Test Reviews. In: Chang SM, Matchar DB, Smetana GW, eds. Methods guide for medical test reviews. Rockville (MD), 2012.

20 SH W. Interim manual for clinical practice guideline development: a protocol for expert panels convened by the office of the forum for quality and effectiveness in health care. Journal of perinatology : official journal of the California Perinatal Association. Rockville, MD: U.S.: Department of Health and Human Services, Public Health Service, Agency for Health Care Policy and Research, 1991.
21 Kuzma-O'Reilly B, Duenas ML, Greecher C, et al. Evaluation, development, and implementation of potentially better practices in neonatal intensive care nutrition. Pediatrics 2003;111:e461-70.

22 Krishnamurthy S, Gupta P, Debnath S, et al. Slow versus rapid enteral feeding advancement in preterm newborn infants 1000-1499 G: a randomized controlled trial. Acta Paediatr 2010;99:42-6.

23 Epelman M, Daneman A, Navarro OM, et al. Necrotizing enterocolitis: review of state-of-the-art imaging findings with pathologic correlation. Radiographics 2007;27:285-305.

24 Siegle RL, Rabinowitz JG, Korones SB, et al. Early diagnosis of necrotizing enterocolitis. AJR Am J Roentgenol 1976;127:629-32.

25 Sántulli TV, Schullinger JN, Heird WC, et al. Acute necrotizing enterocolitis in infancy: a review of 64 cases. Pediatrics 1975;55:376-87.

26 Touloukian RJ. Neonatal necrotizing enterocolitis: an update on etiology, diagnosis, and treatment. Surg Clin North Am 1976;56:281-98.

27 Leonidas JC, Hall RT, Amoury RA. [Critical evaluation of the roentgen signs of neonatal necrotizing enterocolitis]. Ann Radiol 1976;19:123-32.

28 Bell RS, Graham CB, Stevenson JK. Roentgenologic and clinical manifestations of neonatal necrotizing enterocolitis. experience with 43 cases. Am J Roentgenol Radium Ther Nucl Med 1971;112:123-34.

29 Kirks DR, O'Byrne SA. The value of the lateral abdominal roentgenogram in the diagnosis of neonatal hepatic portal venous gas (HPVG). Am J Roentgenol Radium Ther Nucl Med 1974;122:153-8.

30 Seibert JJ, Parvey LS. The Telltale triangle: use of the supine cross table lateral radiograph of the abdomen in early detection of pneumoperitoneum. Pediatr Radiol 1977;5:209-10. 